THE JOURNAL OF CHEMICAL PHYSICS VOLUME 15 , NUMBER 9 SEPTEMBER, 1947

\title{
Restricted Rotator Thermodynamic Properties from the Old Quantum Theory
}

\author{
J. O. HaLford \\ Department of Chemistry, University of Michigan, Ann Arbor, Michigan
}

(Received May 7, 1947)

\begin{abstract}
Accurate partition functions and free energies are calculated for the hindered internal rotator from the old quantum theory based upon the quantization rule of Wilson and Sommerfeld. The description of the system is incomplete and the energy levels may even be incorrectly placed, but the partition functions are surprisingly close to those now accepted as correct. In the course of the development the lowest energy state of the harmonic oscillator turns up in its correct position at the half-quantum level.
\end{abstract}

$\mathrm{H}$ IGH precision can be achieved in calculating the contributions of vibration and pure rotation to thermodynamic properties by methods which omit most of the detail of the energy states of the molecule. Vibrations are taken to be harmonic, interactions are assumed to be negligible, and the contributions are expressed by simple equations involving not more than one molecular constant for each degree of freedom.

Before these simplifications could be used with confidence they had to be justified mathematically or by reference to experimental data. For example, Mayer, Brunauer, and Mayer ${ }^{1}$ derived the energy levels of the unsymmetrical three-dimensional rotator and the free internal rotator of the ethane type from the wave mechanics, and showed that the partition functions could be represented by summations involving only moments of inertia and fundamental constants. Their partition functions proved to be the same as those obtained by the classical statistics. Consequently, it was possible for Kassel$^{2}$ to use the classical method when he developed the general case for the rigid framework with attached symmetrical rotators in extension of the important generalization of Eidinoff and Aston. ${ }^{3}$ Kassel's result proved for practical purposes to be the same as had been predicted by the writer. ${ }^{4}$

Another less sweeping simplification is applicable to the rotator in regions where the classical

\footnotetext{
1 J. E. Mayer, S. Brunauer, and M.G. Mayer, J. Am. Chem. Soc. 55, 37 (1933).

${ }^{2}$ L. S. Kassel, J. Chem. Phys. 4, 276 (1936).

${ }^{3}$ M. L. Eidinoff and J. G. Aston, J. Chem. Phys. 3, 379 (1935).

${ }_{4}^{4}$ J. O. Halford, J. Chem. Phys. 2, 694 (1934).
}

statistics are no longer accurate. Here the energy levels based upon the old quantum theory give the same partition functions as those derived from the wave mechanics. For the harmonic oscillator the old quantum theory may be used in spite of the fact that the energy levels are incorrectly located. Consequently, except for restricted internal rotation, it appears that the finer details of the wave mechanics contribute nothing of practical significance to the ordinary calculation of thermodynamic properties.

In this paper it will be shown that a simplified method may also be used for the internal rotator. The old quantum theory based upon the WilsonSommerfeld quantization rule yields partition functions which do not differ significantly from those obtained by more detailed methods. The description of the energy states is incomplete and the individual levels may be in incorrect positions, but the partition functions and, therefore, the thermodynamic properties always assume values very close to those now accepted as correct.

The old quantum theory handles this problem without any assistance and leads to a minor uncertainty only in the region of close approach to free rotation. The uncertainty in the free energy, however, remains within about 0.02 cal./mole/deg., and only a small and unimportant part of the tables of thermodynamic properties is affected.

Even the half-quantum of energy in the ground state of the harmonic oscillator is revealed correctly by the old quantum theory of the hindered internal rotator. This unexpected result is a consequence of the necessity for establishing a consistent scale of quantum numbers to be used 
in both the vibrational and the rotational regions. Thus one of the major faults of the method disappears during the present application.

It is doubtful if any procedure which had been so successful in its day has ever been more completely discarded than the Wilson-Sommerfeld quantization method. Confidence in its results has reached such a low point that nearly everyone, including the writer, would be unwilling to trust its application to the restricted rotator if tabulations derived by methods accepted as more rigorous were not available for comparison. As far as thermodynamic functions are concerned, the present study indicates that it has not been wise to discard this useful tool so completely.

It becomes possible to calculate the thermodynamic properties of a compound, except in very unusual cases, without recourse to the detail of the wave mechanics. This statement has valuable practical implications, because it is evident that the complications inherent in the solution of the Schroedinger equation can constitute a serious obstacle and may lead the user into making approximations which tend to nullify the advantages of the method.

The effect of varying the assumed potential energy can now be studied in a simpler manner than the one outlined by Pitzer and Gwinn. ${ }^{5}$ There appears to be some theoretical justification for a function of the form

$$
V=\left(V_{0} / 2\right)[1+\cos n \theta+k(1-\cos 2 n \theta)],
$$

which corresponds roughly to curve $C$ in their Fig. 2. Calculations are now in progress to determine whether a detailed study of this function would be justified.

\section{RESULTS}

Table I shows the differences between the free energies of Pitzer and Gwinn and those obtained

TABLE I. Deviation of old quantum theory free energies (cal./mole/deg.) from the results of Pitzer and Gwinn.

\begin{tabular}{rccccccc}
\hline & & & & & & \\
$V / R T T^{1 / Q f} 0$ & 0.1 & 0.2 & 0.4 & 0.55 & 0.60 & 0.80 \\
\hline 0.4 & +0.0007 & -0.004 & -0.003 & +0.002 & -0.003 & & \\
1.0 & -0.0004 & +0.002 & +0.006 & -0.002 & -0.011 & & \\
4.0 & +0.0006 & -0.002 & +0.003 & +0.002 & & +0.005 & \\
9.0 & -0.0002 & 0.000 & -0.001 & 0.000 & & +0.001 & 0.00 \\
16.0 & -0.0003 & -0.001 & -0.001 & 0.000 & & 0.000 & 0.000 \\
\hline
\end{tabular}

${ }^{5}$ K. S. Pitzer and W. D. Gwinn, J. Chem. Phys. 10, 428 (1942). in this study at twenty-seven points distributed over the entire useful range of the variables $V / R T$ and $1 / Q_{r}$. A positive difference means that $F / T$ or $\left(F-F_{f}\right) / T$ is greater than the Pitzer and Gwinn value. $V$ is the height of the simple cosine potential barrier, $R$ the gas constant (1.9869), $T$ the absolute temperature, $Q_{f}$ the partition function of the free rotator,

$$
Q_{f}=2.8148\left(10^{38} I T\right)^{\frac{1}{2}} / n,
$$

and $I$ is the reduced moment of inertia.

The largest difference appears at $1 / Q_{f}=0.55$ and $V / R T=1.0$. At this point an adjustment of the zero state to insure asymptotic approach to the free rotator, as described later, becomes most uncertain, but the spread of reasonable adjustments should not cause deviations larger than 0.02 in the column for $1 / Q_{f}=0.55,0.015$ for $1 / Q_{f}=0.50$, and 0.01 for $1 / Q_{f}=0.45$. Within each column, it has been found by calculations not included in the table that the deviations decrease rapidly from the maximum as $V / R T$ is changed in either direction.

For further application of the old quantum theory it would be permissible to adjust the values in this region toward those of Pitzer and Gwinn. For the present, however, they are left as they are in order to show what the method produces without assistance from other sources. In any case the deviations are unimportant in relation to the errors of thermodynamic data, the arbitrary selection of the potential function and the probability that the region of the largest deviations will not be used unless methyl alcohol proves to have an unexpectedly low potential barrier. $^{6}$

It is obvious without further calculation that the other thermodynamic properties will be close to those found by Pitzer and Gwinn.

\section{ENERGY LEVELS}

The total energy $E$ is expressed as the sum of the kinetic and potential energies by the equation

$$
2 E=I \dot{\theta}^{2}+V(1-\cos n \theta),
$$

in which $\theta$ is a rotational coordinate and $n$ is the symmetry number or the number of potential minima in the cycle. The momentum $p$, equal to

\footnotetext{
${ }^{6}$ J. O. Halford, J. Chem. Phys. 15, 364 (1947).
} 
$I \dot{\theta}$, becomes

$$
\begin{aligned}
& p=I^{\frac{1}{2}}[2 E-V(1-\cos n \theta)]^{\frac{1}{2}} \\
& =(I V)^{\frac{1}{2}}[2(E / V-1)+(1+\cos n \theta)]^{\frac{1}{2}} .
\end{aligned}
$$

For states with energy less than $V, E / V-1$ is equal to $-\left(1+\cos n \theta_{0}\right) / 2$, where $\theta_{0}$ is the amplitude of vibration.

The Wilson-Sommerfeld rule requires that

$$
\begin{aligned}
r h=\oint p d \theta=(I V)^{\frac{1}{2}} \oint[2(E / V-1) \\
+(1+\cos n \theta)]^{\frac{1}{2}} d \theta
\end{aligned}
$$

with $r$ to have only integral values. The symmetry number can be factored out of the integral by the substitution $\varphi=n \theta$. Limits are then assigned for a complete cycle in $\varphi$ to give

$$
\begin{aligned}
r h=2(I V)^{\frac{1}{2}} / n \int_{0}^{\pi}[2(E / V+1) & \\
& +(1+\cos \varphi)]^{\frac{1}{2}} d \varphi .
\end{aligned}
$$

This equation states that the rotational quantum number at a given total energy is one $n$th of the quantum number for the same energy in the single minimum case and means that only each $n$th rotational doublet is to be counted. The corresponding point in the Pitzer and Gwinn development is the assignment of one state to each of the regions delimited by their theory. In Eq. (5), for convenience, the integration is performed over half the cycle and the result is multiplied by two.

The quantum number obtained in this manner gives the mean position of the rotational doublet. If the integration is performed in the vibrational region strictly according to the rule, the upper limit is changed to the amplitude, $\varphi_{0}$, and the length of the cycle is doubled. Here, to keep rotations and vibrations on the same number scale, the doubling is omitted and the rotational scale is retained into the vibrational region, where each quantum number $r$ now gives the mean position of two vibrational states. But in the harmonic oscillator limit the individual states are equally spaced on both the energy and number scales. The two number scales, vibrational and rotational, become consistent if the vibrational states are placed at $r=1 / 4,3 / 4,5 / 4$, $\cdots$, corresponding to the vibrational quantum numbers $1 / 2,3 / 2,5 / 2, \cdots$. In this way the harmonic oscillator acquires an energy $h \nu / 2$ in the ground state by an argument based upon the Wilson-Sommerfeld quantization rule.

The key to the use of the old quantum theory in the internal rotation problem is to retain under all conditions the rule that the rotational quantum number $r$ is the arithmetical mean of the quantum numbers of the two components of the doublet.

The manner of arriving at Eq. (5) suggests that it should give a solution for the energy levels in the single minimum case when there is no over-all rotation of the molecule around the axis of the internal motion. This interpretation is in qualitative agreement with diagrams published by Koehler and Dennison ${ }^{7}$ for the methanol case in illustration of their general solution for the energy levels with the simple cosine potential function.

For states entirely in the rotational region the theory fails to designate the doublet separation. This is known, however, at the top of the barrier, and can be reasonably assumed to approach zero for high energy levels, a situation that can be approximately represented by assigning the positions $r \pm V / 4 E$. Because of a tendency toward cancellation of the errors introduced by this assumption, its effect is felt only in those cases where the first doublet is at a short distance above the top of the barrier.

The integration of Eq. (5) has been performed graphically at chosen values of $\varphi_{0}$ or $E / V$. At

TABLE II. Energy of the internal rotator in terms of the quantum number index.

\begin{tabular}{lcl}
\hline$E / V$ & $A$ (radians) & $\rho$ \\
\hline 0.03016 & 0.06724 & 0.008539 \\
0.1170 & 0.26385 & 0.033505 \\
0.2500 & 0.5746 & 0.07297 \\
0.4132 & 0.9749 & 0.12348 \\
0.5868 & 1.4305 & 0.18165 \\
0.7500 & 1.9005 & 0.24134 \\
0.8830 & 2.3344 & 0.29644 \\
0.9700 & 2.6729 & 0.3394 \\
1.00 & 2.8284 & 0.3592 \\
1.10 & 3.2550 & 0.4133 \\
1.25 & 3.7267 & 0.4732 \\
1.50 & 4.3689 & 0.5548 \\
2 & 5.4026 & 0.6861 \\
3 & 7.0071 & 0.8898 \\
5 & 9.4175 & 1.1959 \\
9 & 12.9503 & 1.6445 \\
\hline
\end{tabular}

7 J. S. Koehler and D. M. Dennison, Phys. Rev. 57, 1006 (1940). 
TABLE III. $\delta$ (Eq. (9)) as a function of $\rho$.

\begin{tabular}{cc}
\hline$\rho$ & $\delta$ \\
\hline 0.008539 & 0.00002 \\
0.033505 & 0.00024 \\
0.07297 & 0.00098 \\
0.12378 & 0.00215 \\
0.18165 & 0.00235 \\
0.24134 & 0.00000 \\
0.29644 & -0.00863 \\
\hline
\end{tabular}

each point from twenty to forty ordinates were calculated and the area was approximated for the separation $\Delta \varphi$ and the ordinates $y_{0}, y_{1}, \cdots$, $y_{n}$, according to the equation

$$
A=\Delta \varphi\left[\left(y_{0}+y_{n}\right) / 2+y_{1}+\cdots+y_{n-1}\right] \text {. }
$$

Apparent areas $A$ for several values of $\Delta \varphi$ were plotted against $\Delta \varphi$ and extrapolated to $\Delta \varphi=0$. For this purpose, if the number of ordinates is chosen conveniently, only one addition is necessary. For example, with forty ordinates the first total is based upon $y_{0}, y_{8}$, etc. To this are added $y_{4}, y_{12}$, etc., to give the second total for half the spacing first used. The rest of the process is evident. Simpson's one-third rule might have been used, but the writer is a little suspicious of it and preferred the method just described. In a few tests the error made with Simpson's rule for a given $\Delta \varphi$ proved to be about equal to that made with Eq. (6) for $\Delta \varphi / 2$. Any advantage of Simpson's rule is partly offset by the extra work of adding the ordinates in new combinations and the need for enough ordinates to provide three points for the extrapolation.

As the ratio $E / V$ increases above 1.0 the area rapidly approaches that of a rectangle erected on the ordinate at $\varphi=\pi / 2$ and can be obtained accurately with quite large spacing of the ordinates. At the top of the barrier the integration is carried out analytically to give an area of $2^{\text {s }}$ radians and provide a check on the accuracy of the graphical method. The difference appears in the fifth digit beyond the decimal point. Incidentally the classical picture of a vibration at this point is rather amusing. At the maximum amplitude the restoring force is zero, suggesting that the period should be infinite and the frequency zero.

Equation 5 is combined with the equation

$$
Q_{f}=\left(8 \pi^{3} I k T\right)^{\frac{3}{3}} / n h
$$

to give Eq. (8), in which $\rho$ is a convenient quantity that may be called the quantum number index.

$$
r /\left[Q_{f}(V / R T)^{\frac{1}{2}}\right]=A /\left(2 \pi^{3}\right)^{\frac{1}{2}}=\rho .
$$

Since the relation between $E / V$ and $A$ or $\rho$ is the same for all systems within the range of the variables a single curve or its equivalent in tables and equations will summarize the results of the quantization.

Table II shows in successive columns the related values of $E / V$, the integral $A$, and the quantum number index $\rho$.

The information in Table II can be expressed in equations to be used with tables of differences. For $E / V$ between zero and 0.75 the equation is

$$
E / V=3.545 \rho-1.812 \rho^{2}+\delta \quad(\rho<0.24),
$$

for which $\delta$ is shown in Table III.

At higher levels the equation

$$
E / V=\pi \rho^{2}+0.5+\Delta \quad(\rho>0.24)
$$

is valid. The values of $\Delta$ appear in Table IV. For interpolation in Tables III and IV it is convenient to use the graphical method.

It is evident that the curve of $E / V$ against $\rho$ is asymptotic to the harmonic oscillator line $E / V=2 \pi^{\frac{3}{2}} \rho$ in the low energy limit, and approaches the parabola $E / V=\pi \rho^{2}+0.5$ at high energies. The latter limit is consistent with the statement by Koehler and Dennison that the high levels approach the free rotator energies plus $V / 2$. The relation to the harmonic oscillator and the limiting rotator is illustrated in Fig. 1.

In terms of the variables used here the equations for the harmonic oscillator and the free rotator take particularly simple forms. For the oscillator the expressions $E=2 r h \nu$ and

TABLE IV. $\Delta$ (Eq. (10)) as a function of $\rho$.

\begin{tabular}{cc}
\hline$\rho$ & \multicolumn{1}{c}{$\Delta$} \\
\hline 0.1817 & -0.01683 \\
0.2413 & +0.06703 \\
0.2964 & 0.10695 \\
0.3394 & 0.10792 \\
0.3592 & 0.09471 \\
0.4133 & 0.06325 \\
0.4732 & 0.04641 \\
0.5548 & 0.03303 \\
0.6861 & 0.02132 \\
0.8898 & 0.01259 \\
1.1959 & 0.00695 \\
1.6445 & 0.00371 \\
\hline
\end{tabular}


$\nu=n(V / 2 I)^{\frac{1}{2}} / 2 \pi$ are combined with Eqs. (7) and (8) to give $E=2 \pi^{\frac{2}{2}} \rho V$. For the free rotator, the relation $E=(n r h)^{2} / 8 \pi^{2} I$ together with Eqs. (7) and (8) yields $E=\pi \rho^{2} V$. Although $\rho$ becomes infinite as $V$ approaches zero, the energy remains definite for all finite values of the quantum number $r$.

To find the allowed energies for a given rotator, the quantum numbers $r \pm \frac{1}{4}$ and $r \pm V / 4 E$, where $r$ is an integer, are divided by the product $Q_{f}(V / R T)^{\frac{1}{2}}$ (which is independent of the temperature) to give the required values of the index $\rho$, after which the energy ratios $E / V$ are determined from Eqs. (9) and (10) and Tables III and IV. The sum of the quantities $e^{-\left(E / V-E_{0} / V\right)(V / R T)}$ is then the required partition function.

This simple procedure gives highly accurate partition functions and free energies for all cases for which three or more individual energy levels lie within the potential valley, and covers practically all situations for which the thermodynamic properties would probably be needed. The special treatment used between this point and the free rotation limit is considered in a separate section.

\section{HIGH TEMPERATURE LIMIT}

When $1 / Q_{f}$ approaches zero the spaçing of the energy states becomes infinitesimal and thepartition function may be expressed by means of the following integral:

$Q=2 \int_{0}^{\infty} e^{-E / R T} d r=2 Q_{\delta}(V / R T)^{\frac{1}{2}} \int_{0}^{\infty} e^{-E / R T} d \rho$.

As Pitzer $^{8}$ originally found, the ratios $Q / Q_{f}$ and the corresponding free energy differences are finite, although the partition functions themselves are not.

The integral from $\rho=0$ to $\rho=0.24$ was found by the graphical method in the manner described above in connection with the quantizations. The remainder of the integral was evaluated closely enough by a rapid procedure which enlisted the aid of tabulated values of the probability integral

$$
P_{1}=\left(2 / \pi^{\frac{1}{2}}\right) \int_{0}^{x_{1}} \exp \left(-x^{2}\right) d x,
$$

\section{found in Mellor's Higher Mathematics. ${ }^{9}$}

\footnotetext{
${ }^{8}$ K. S. Pitzer, J. Chem. Phys. 5, 469 (1937).

$9 \mathrm{~J}$. W. Mellor, Higher Mathematics for Students of Chemistry and Physics (Dover Publications, New York, 1946), p. 621 .
}

The integral between the limits $x_{1}$ and $x_{2}$ is $P_{2}-P_{1}$, and for our purposes takes the form

$$
P_{2}-P_{1}=\left(2 a / \pi^{\frac{1}{2}}\right) \int_{a_{\rho 1}}^{a \rho \rho 2} \exp \left[-a^{2} \rho^{2}\right] d \rho
$$

from which, if $a=(\pi V / R T)^{\frac{1}{3}}$, the fraction of the partition function of a free rotator which comes from the region between $\rho_{1}$ and $\rho_{2}$ is given directly by $P_{2}-P_{1}$ as found in the table for $x_{1}=a \rho_{1}$ and $x_{2}=a \rho_{2}$. If $\rho_{1}$ and $\rho_{2}$ are not far apart, the correction $\Delta$ of Table IV may be regarded as constant, and the contribution of the region to $Q / Q_{f}$ will be given by

$$
\left[Q / Q_{f}\right]_{\rho 1}^{\rho 2}=\left(P_{2}-P_{1}\right) e^{-(0.5+\Delta) V / R T} .
$$

As the energy ratio approaches the limit $E / V=\pi \rho^{2}+0.5$, the fractions may be taken over increasingly wide intervals. The result is nearly as accurate as the direct graphical integration when only twelve areas are summed in this manner. The procedure is equivalent to replacing the $E / V$ curve by a series of parabolic sections parallel to the free rotator curve before performing the integration.

\section{APPROACH TO FREE ROTATION}

In the harmonic oscillator limit the ground state is found at the position $r_{0}=\frac{1}{4}$ or $\rho_{0}=1 /\left[4 Q_{f}(V / R T)^{\frac{1}{2}}\right]$. If this relation were applied in the region of close approach to free rotation,

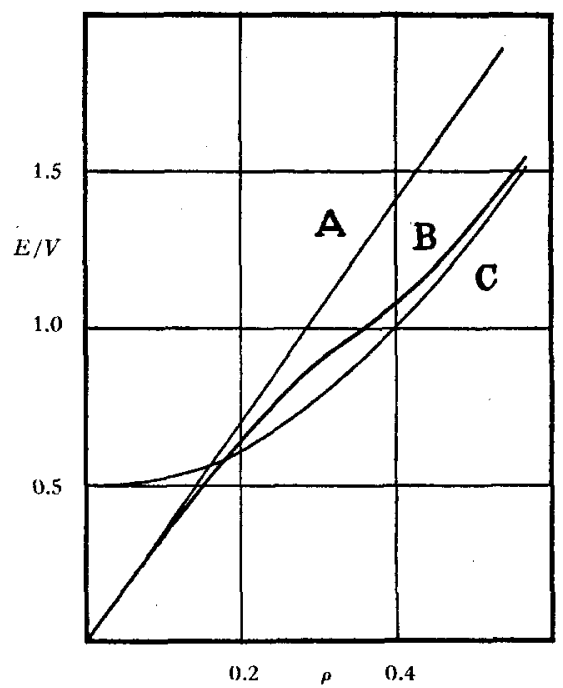

Fig. 1. Ratio of energy to barrier height as a function of the rotational quantum number index. $A$, harmonic oscillator. $B$, restricted rotator. $C$, limiting rotator. 
TABLE V. Energy levels of methyl alcohol for $V=770 \mathrm{~cm}^{-1}$.

\begin{tabular}{ccccc}
\hline \multicolumn{2}{c}{$E / V(K$ and $D$ ) } & & & $\begin{array}{c}E / V \\
\text { Min. }\end{array}$ \\
Max. & $r$ & $\rho$ & (this study) \\
\hline 0.2482 & 0.2506 & $\mathbf{0 . 2 5}$ & $\mathbf{0 . 0 7 5 9 4}$ & 0.2598 \\
0.6784 & 0.7187 & $\mathbf{0 . 7 5}$ & $\mathbf{0 . 2 2 7 8 2}$ & 0.7144 \\
0.9644 & 1.1697 & 1.25 & $\mathbf{0 . 3 7 9 7 0}$ & 1.0329 \\
1.2152 & 1.6766 & 1.85 & $\mathbf{0 . 5 6 1 9 6}$ & 1.5241 \\
\hline
\end{tabular}

with $Q_{f}$ constant and $V / R T$ approaching zero, the zero state would appear to emerge from the potential valley at a finite value of $V / R T$, and the ratio $E_{0} / V$ would increase indefinitely as the barrier decreased toward zero. It is easily shown that in the limit the energy would be onesixteenth that of the first rotational doublet, and that when the zero state was above $V / 2$, the partition function would exceed the free rotation value.

It is, therefore, necessary to adjust the zero state downward if an asymptotic approach to free rotation is to be achieved. While it is obvious that the zero-point energy must disappear in the limit, there is no evident reason why the partition function should not exceed the free rotation value through some limited range of very low potential barriers.

An examination of the graphical illustrations of Koehler and Dennison raises the same question in connection with the region over which the zero state may roam in the triple-minimum problem as the potential barrier decreases toward zero. The answer would come from a detailed study of the combined partition function of the coupled internal and external rotations, and will be sought at some future date when time permits.

For the present it is assumed that the zero state will remain low enough to insure an asymptotic approach of the partition function to the free rotator limit from lower values. An adjustment is made according to the arbitrary relation

$$
\alpha \rho_{0}{ }^{\beta}+\rho_{0}=1\left[4 Q_{f}(V / R T)^{\frac{1}{2}}\right]=\rho_{1} / 4 .
$$

There appears to be no way to determine $\alpha$ and $\beta$ uniquely, but satisfactory evaluations can be made by considering the behavior of the function in two limiting regions. It is required, if $r$ is to be at the mean position of the doublet, that the first term of Eq. (15) shall become negligible when the first doublet is entirely within the potential valley, where the separation is fixed by the quantization over the vibrational cycle. For this situation $\rho_{0}$ is 0.072 , and an error of about 0.0004 can be made without introducing a significant uncertainty into the partition function. If $\beta$ is assigned, the corresponding $\alpha$ is determined by this assumed error. The behavior of the function near free rotation for various values of $\beta$ is next examined. It is assumed that at $1 / Q_{f}=0.55$ and $V / R T=0.10$ the partition function will be below and very close to the free rotator value, and the lowest integral value of $\beta$ is taken for which this is true. The equation then takes the form

$$
7.7 \times 10^{6} \rho_{0}{ }^{9}+\rho_{0}=\rho_{1} / 4 .
$$

Actually the deviations in Table I are smaller for the next higher power of $\rho_{0}$. A change in the permissible uncertainty at $\rho_{0}=0.072$ will alter the selection of $\alpha$ and $\beta$ without producing much change in the size of the corrective term. The procedure is quite arbitrary, but no reasonable change in the assumptions produces serious differences in the partition functions and free energies.

For $\rho_{1}$ greater than 0.29 , Eq. (16) is used to . find the position of the ground state. If the energy at $r_{0}+1 / 2$ falls within the barrier, the lower component of the first doublet is placed at this point and the second component is moved upward to $3 / 2-r_{0}$ in order to keep $\rho_{1}$ for $r=1$ at the mean position. When $r_{0}+1 / 2$ is in the rotational region the doublet components are placed at $r=1 \pm V / 4 E$.

As might be expected, the largest deviations of Table I occur near the midpoint, on the $\rho_{0}$ scale, of the region spanned by Eq. (16).

\section{METHYL ALCOHOL}

The energy levels given by the old quantum theory can be compared with those calculated by Koehler and Dennison for methyl alcohol with an assumed potential barrier of $769.43 \mathrm{~cm}^{-1}$. They have described the first four levels in detail, showing exactly how the components of each triplet change in position with the quantum number of the external rotation about the axis of the methyl top. In Table $V$, the first and second columns show the ratio $E / V$ for the 
minimum and maximum positions of the states within each triplet, and the third, fourth, and fifth give the values of $r, \rho$, and $E / V$ obtained in this study. The reduced moment of inertia was calculated from other constants in their paper to be $1.116 \times 10^{-40}$.

At the upper levels the old quantum theory result appears alternatively toward the upper or lower end of the region delimited by the wave mechanics. At the same end of each region, when the external quantum number is zero, is found a component of a separated doublet derived from a rotational state whose quantum number in the complete system of levels is a multiple of 3 , or, more generally, a multiple of $n$. The Wilson-Sommerfeld rule, therefore, gives an average position weighted toward the true level taken by a component of each $n$th state when there is no external rotation. The upward displacement of the odd numbered states, on the vibrational scale of quantum numbers, is greater than the downward displacement of the even numbered states.

For the same zero level the old quantum theory would lead to a lower partition function than the one obtained from the mean positions of the regions derived from the wave mechanics. In Table V, however, the zero state is a little high, and our experience indicates that it is just high enough in general to compensate for the different description of the higher levels and lead to the correct partition functions.

THE JOURNAL OF CHEMICAL PHYSICS VOLUME 15, NUMBER 9 SEPTEMBER, 1947

\title{
Statistical Properties of Networks of Flexible Chains
}

\author{
HuberT M. JAMES \\ Purdue University, West Lafayette, Indiana
}

(Received May 12, 1947)

A Gaussian network is defined as a network of flexible chain segments, linked to each other and to a system of fixed points, in which each unbranching chain segment can take on a number of configurations which is a Gaussian function of the distance between its ends. Real molecular networks, such as those of rubber, can under certain circumstances be treated as Gaussian networks. The present paper carries out a systematic mathematical discussion of the statistical properties of Gaussian networks: the total number of possible configurations of the network as a function of the fixed-point coordinates, the probability of finding a given element of the network in a given position, or of finding two elements of the network in given relative positions, and so on. All probability-density functions

$\mathbf{R}$ UBBER-LIKE materials consist of molecular networks formed by the random bonding together of long flexible molecular chains. ${ }^{1}$ In developing the theory of rubber-like elasticity it is necessary to study the statistical properties of these networks-to determine how the total numbers of configurations possible for the network depends on the external constraints,

\footnotetext{
${ }^{1}$ H. M. James and E. Guth, J. Chem. Phys. 11, 455 (1943).
}

appear as exponentials of quadratic forms, with constants explicity expressible in determinant form. An explicit reduction to a sum of squares is given for all quadratic forms occurring in the theory of coherent Gaussian networks, and an explicit general formula is found for integrals of the form

$$
\int_{-\infty}^{+\infty} d X_{1} \int_{-\infty}^{+\infty} d X_{2} \cdots \int_{-\infty}^{+\infty} d X_{q} \exp \left\{-\sum_{i}^{n} \sum_{i}^{n} \gamma_{i j} X_{i} X_{j}\right\}
$$

There is described a mechanical analog of a Gaussian network, by consideration of which the statistical properties of the Gaussian network can be determined. The method is applied to the discussion of the statistical properties of a Gaussian network with the connectivity of a regular cubic lattice.

to compute the probability that a given element of the network is in a given position, or that a given pair of elements are in given relative positions, and so on. It has been shown elsewhere ${ }^{1}$ that for such purposes it is a reasonable approximation (in the case of soft rubber) to assume that each unbranched chain segment in the network can take on a number of configurations which is a Gaussian function of the separation of its ends, and this independently of the 